\title{
Self-oscillating Gel Accelerated while Sensing the Shape of an Aqueous Surface
}

Miyu Yoshii ${ }^{1}$, Hiroya Yamamoto ${ }^{1}$, Yutaka Sumino ${ }^{2}$, and Satoshi Nakata ${ }^{1,3, *}$

1. Graduate School of Science, Hiroshima University, 1-3-1 Kagamiyama,

Higashi-Hiroshima, Hiroshima 739-8526, Japan

2. Department of Applied Physics, Faculty of Science, Tokyo University of Science,

6-3-1 Niijuku, Katsushika, Tokyo 125-8585, Japan

3. Research Center for the Mathematics of Chromatin Live Dynamics, Japan

Supporting Information (SI)

Hiroshima Univ. 


\section{Movies of reciprocating motion in Figs.3, 4, and 5}

Movie S1. Movie of reciprocating motion in Fig. 3 (video rate: 50 times as fast as the actual speed).

Movie S2. Movie of reciprocating motion in Fig. 4 (video rate: 50 times as fast as the actual speed).

Movie S3. Movie of reciprocating motion in Fig. 5 (video rate: 100 times as fast as the actual speed).

\section{Periodic motion of a self-oscillating gel on a sloped surface}

We observed the periodic reciprocating motion (Mode II) of the gel on a sloped surface in the concave geometry (Water chamber: the glass Petri dish $(I D=16 \mathrm{~mm})$ ). 

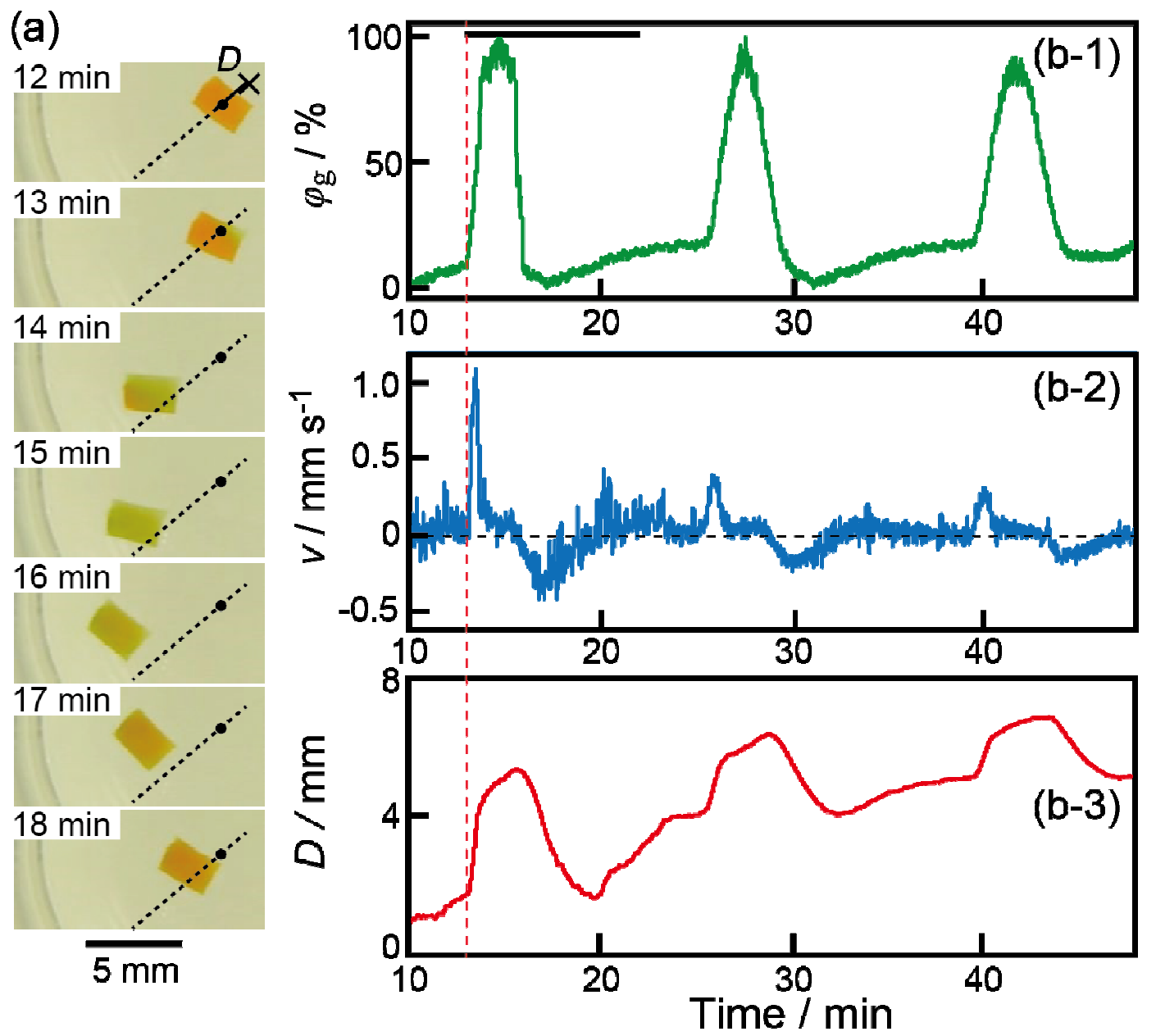

Figure S1. (a) Snapshots of a gel on a sloped surface (top view), (b) time-course of (b-1) the percentage of green area, $\varphi_{\mathrm{g}},(\mathrm{b}-2)$ the velocity of the gel on the BZ solution, $v$, and (b-3) the distance between the center of the Petri dish $(\times)$ and that of the gel, $D$ (Mode II). ID = $16 \mathrm{~mm}$ (Fig. 1a). The horizontal bar in (b-1) corresponds to the timespan shown as the snapshots in (a). The dotted line in (a) is drawn from the center to a wall of the Petri dish via the center location of the gel at $t=12 \mathrm{~min}$. Definition of the $v$ is the same as that in Fig. 3. The red dotted line denotes the time at $v=0$.

\section{Quantitative estimation of forces acting on the gel}


The forces acting on the gel, $F_{\mathrm{s}}$ and $F_{\mathrm{c}}$ are estimated from eqs 2 and 5 .

Contact angle for reduced state, $\theta_{\text {red, }}$ is $48 \mathrm{rad}$, and for oxidation state, $\theta_{\mathrm{ox}}$, is $42 \mathrm{rad}$ from our previous report. ${ }^{24}$ From eq 2, we can estimate the maximum size of $F_{\mathrm{s}}$ as, $F_{\mathrm{s}}=F_{\mathrm{L}}-F_{\mathrm{R}}=l_{\mathrm{w}} \gamma_{\mathrm{aw}}\left(\sin \theta_{\mathrm{L}}-\sin \theta_{\mathrm{R}}\right)=0.002 \mathrm{~m} \times 0.040 \mathrm{~N} / \mathrm{m} \times(0.743-0.669)=6 \mu \mathrm{N}$, where we have used $\theta_{\mathrm{red}}$ for $\theta_{\mathrm{L} \text {, and }} \theta_{\mathrm{ox}}$ for $\theta_{\mathrm{R}}$. From the measured water level shown in Fig.1, we can obtain $d z / d x=0.149$ for the gel whose position is at $D=2.3 \mathrm{~mm}$ and front position is $x=4.3$ (Petri dish: $I D=16 \mathrm{~mm}$ ). Thus, from eq 5, we can estimate $F_{\mathrm{c}}$ for fully reduced state, $F_{\mathrm{c}, \text { red }}$ and for fully oxidation state $F_{\mathrm{c}, \text { red }}$ as,

$$
\begin{aligned}
& F_{\mathrm{c}, \mathrm{red}}=\gamma_{\mathrm{aw}} \frac{d z}{d x} \int \cos \theta(l) d l=0.040 \mathrm{~N} \mathrm{~m}^{-1} \times 0.149 \times 0.669 \times 0.008 \mathrm{~m}=32 \mu \mathrm{N}, \text { and } \\
& F_{\mathrm{c}, \mathrm{ox}}=\gamma_{\mathrm{aw}} \frac{d z}{d x} \int \cos \theta(l) d l=0.040 \mathrm{~N} \mathrm{~m}^{-1} \times 0.149 \times 0.743 \times 0.008 \mathrm{~m}=35 \mu \mathrm{N} .
\end{aligned}
$$

